\title{
Entropy-based Abnormal Activity Detection Fusing RGB-D and Domotic Sensors
}

\author{
Manuel Fernandez-Carmona, Serhan Cosar, Claudio Coppola, Nicola Bellotto*
}

\begin{abstract}
The automatic detection of anomalies in Active and Assisted Living (AAL) environments is important for monitoring the wellbeing and safety of the elderly at home. The integration of smart domotic sensors (e.g. presence detectors) and those ones equipping modern mobile robots (e.g. RGB-D cameras) provides new opportunities for addressing this challenge. In this paper, we propose a novel solution to combine local activity levels detected by a single RGB-D camera with the global activity perceived by a network of domotic sensors. Our approach relies on a new method for computing such a global activity using various presence detectors, based on the concept of entropy from information theory. This entropy effectively shows how active a particular room or environment's area is. The solution includes also a new application of Hybrid Markov Logic Networks (HMLNs) to merge different information sources for local and global anomaly detection. The system has been tested with a comprehensive dataset of RGB-D and domotic data containing data entries from 37 different domotic sensors (presence, temperature, light, energy consumption, door contact), which is made publicly available. The experimental results show the effectiveness of our approach and its potential for complex anomaly detection in AAL settings.
\end{abstract}

\section{INTRODUCTION}

One of the current challenges in healthcare systems is the increasing life-expectancy [1]. Longer life-expectancy implies bigger social and economic burdens to cope with chronic illnesses. Improving the quality of life through AAL technologies and promoting independent living at home for elderly people can ease these effects.

People with Mild Cognitive Impairment (MCI) are functionally independent, except for occasional episodes of disorientation, memory loss or cognitive difficulty [2]. The EU project ENRICHME ${ }^{1}$ focuses on developing new AAL technologies to enable health monitoring, complementary care and social support for elderly people with MCI, helping them to remain active and independent for longer. An important task of the project is the detection of unusual human activities, which are known to be an early indicator of dementia. For example, they are highly correlated with the sundown syndrome, which is manifested through abnormal motor activity levels, particularly in the afternoon [3].

Domotic sensors are a particularly attractive technology for activity monitoring tasks: they are typically cheap, pervasive and unintrusive. These features make them relevant for the detection of activity levels in AAL. However, due to their low consumption and bandwidth, they can only

\footnotetext{
*Authors are with Lincoln Centre for Autonomous Systems (L-CAS), University of Lincoln, LN6 7TS, UK \{mfernandezcarmona, scosar, ccoppola, nbellotto \}elincoln.ac.uk

${ }^{1}$ ENabling Robot and assisted living environment for Independent Care and Health Monitoring of the Elderly - http://www.enrichme.eu
}

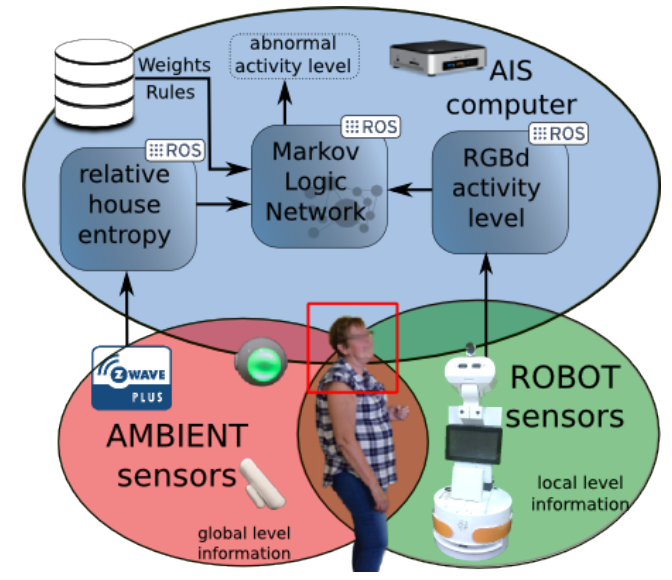

Fig. 1: Robot \& domotic sensors integration in ENRICHME.

provide a limited amount of information with low resolution (i.e. motion inside a room). These limitations could be solved using more expensive but also data-rich sensors, like the RGB-D camera equipping many of nowadays robots, including the one in ENRICHME (see Fig 1). This sensor provides far more accurate, although localized, information.

The challenge is how to effectively combine these different sensor sources in order to detect abnormal activity levels. To this end, the current paper includes three main contributions:

- a new entropy-based metric to detect human activity with domotic sensors, which does not require complex training or manual data labelling;

- a new application of HMLN to combine different source of information for anomaly detection, merging categorical data from a local (RGB-D) activity detector with the continuous entropy-based one from global (domotic) sensors;

- a large public dataset, containing data entries from 37 different domotic sensors (presence, temperature, light, energy consumption, door contact).

The remainder of the paper is organized as follows. Sec. II describes relevant state-of-the-art approaches for anomaly detection with domotic sensors. The integrated system of domotic sensors and RGB-D camera is presented in Sec. III. Local and global activity level detectors are explained in Sec. IV. The following Sec. V describes the Markov Logic Network (MLN) implemented to combine the two activity level detectors and discover potential anomalies. The dataset used for the experiments and the results are presented in Sec. VI. Finally, the outcomes and limitations of this approach, as well as future work, are discussed in Sec. VII. 


\section{RELATED WORK}

Perhaps, the most obvious choice for anomaly detection relies on wearable sensors, such as activity trackers. Godfrey et al. [4] developed an application to detect symptoms of the sundown syndrome using these sensors. Unfortunately, this approach is not always suitable for MCI patients as they may forget or just refuse to wear such devices. Another possible choice is to analyse video sequences. $\mathrm{Xu}$ et al. [5] presented a method to predict anomalies based on multiple one-class Support Vector Machine (SVM) models, offering an anomaly score for video sequences. In [6], instead, an RGB-D camera was used to detect Activities of Daily Living (ADLs) using Hidden Markov Models.

Domotic sensors are an alternative, usually cheaper choice for anomaly detection. For example, the authors in [7] used domotic sensors to track habits based on length, frequency and periodicity of activity sequences. They developed an activity recognition algorithm, called Episode Discovery model, and proposed anomaly analysis based on this paradigm for their future work. In [8], the authors used presence detectors to build a spatio temporal behaviour model. An anomaly in this context is a significant deviation from the model, detected using a cross-entropy metric. Their model however is very sensitive to potential misplacements or faults of the deployed sensors.

Fusing various sensing modalities can increase the robustness of the system and tolerance to occasional data errors. Though there are a plethora of Bayesian sensor fusion models, such as Kalman filters and similar, these are usually inappropriate for estimating discrete labels or processes where the error characteristics are not easily parametrised [9]. MLNs do not have this limitation. In addition, HMLNs are capable to perform inferences mixing information from both continuous and discrete domains [10].

MLNs are able to perform inference using incomplete inputs [11]. This is a common issue in domotics sensors, where missing datagrams and faulty sensors are frequent problems. Chahuara et al. [12] used a MLN for activity recognition with domotic sensors and microphones in a real scenario. However, they only used qualitative data obtained from pre-processed continuous parameters, instead of exploiting the capability of MLNs to deal with continuous information directly.

Besides MLNs, other statistical approaches were proposed, for example using Case-Based Reasoning (CBR) and fuzzy logic to detect anomalies with domotic and physiological sensors [13]. They also used fuzzy logic to encode additional rules and detect unusual behaviours. Although an interesting system, so far this has only been tested in simulations.

Public datasets of labelled sensor data are important for the research community to test and compare different algorithms. To our knowledge, there are only a few interesting domotic datasets, in particular the one hosted by Tim van Kasteren ${ }^{2}$, and the CASAS extensive collection of datasets ${ }^{3}$. However,

\footnotetext{
${ }^{2}$ https://sites.google.com/site/tim0306/datasets

${ }^{3}$ Center for Advanced Studies in Adaptive Systems - http://ailab. wsu.edu/casas/datasets/index.html
}

usually they are provided as plain text or raw Matlab files and are difficult to handle, especially if of large size. Our publicly available dataset, instead, was created by systematically storing raw data in standard MongoDB format.

\section{SySTEM DESCRIPTION}

The activity monitoring system in ENRICHME exchanges information between the domotic sensor network and the robot (see Fig. 1) through the Ambient Intelligence Server (AIS). The latter consists of an embedded high-specs PC, located at home, which acts as a multiprotocol gateway, collecting and forwarding the information shared by the robot and other smart sensors in the environment.

The AIS is connected via wireless to a set of domotic sensors for motion, contact, and energy consumption monitoring. The system is based on the OpenHAB middleware ${ }^{4}$, which provides an abstraction layer for these sensors [14]. OpenHAB supports a wide range of different domotic technologies with a uniform interface, decoupling sensor information from specific domotic protocols or manufacturers. It also provides an interface and intercommunication ${ }^{5}$ with the Robot Operating System (ROS) network, independently from the ambient sensors actually used.

The domotic network available in the ENRICHME's homes is Z-Wave. It is a wireless mesh network with several different models of sensors. In general, these wireless domotic devices are relatively cheap and easy to deploy within a user home network. Three particular sensors are available in our system: contact detectors, energy meters and multi-parameter sensors. The latter provide information about human presence, light and temperature. Contact sensors instead are used to monitor door states (open, close), while energy sensors are used to monitor the use of relevant electric appliances, such as microwave, coffee machine, etc.

The fact of being physically located in the domestic environment facilitates the communication of the AIS with the robot and the smart sensors, avoiding potential problems due to internet bandwidth or poor quality-of-service. It also provides the opportunity to extend the system with further optional wired sensors (e.g. fixed RGB-D camera) directly connected to the AIS computer.

Domotic sensors are usually fixed, preventing sensing reconfigurations once the system is running. Also, they only provide limited information, which could be insufficient to identify potential anomalies. We use therefore a mobile $\operatorname{robot}^{6}$ (see Fig. 1), equipped with an RGB-D camera and other sensors, as an extension of the AAL environment, which provide some additional (localized) sensing information to overcome these limitations.

The robot can navigate in the domestic environment, monitoring and interacting with the user to offer various services, such as reminding appointments, suggesting exercises, or finding lost objects. Using its sensors (RGB-D

\footnotetext{
${ }^{4}$ http: / / www . openhab.org/

5 https://github.com/corb555/iot_bridge

${ }^{6}$ http: //tiago.pal-robotics.com/
} 


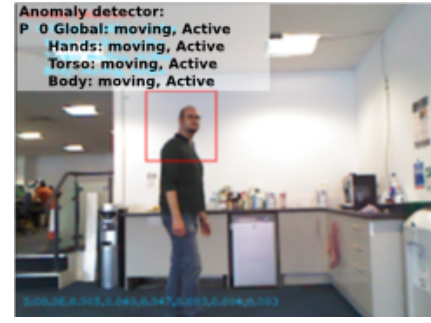

(a)

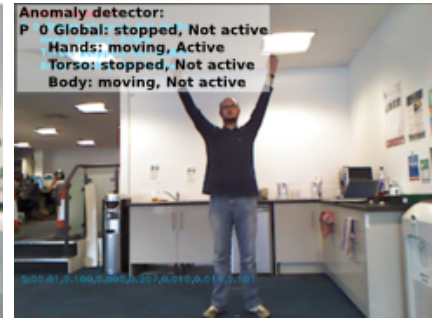

(b)
Fig. 2: Results of activity level detection when the person is (a) walking and (b) moving his arms.

and thermal cameras, 2D laser, RFID antenna), it estimates and shares with the AIS real-time information about the user, including location, activity levels, physiological state, etc., as well as other details for scheduled events and remote connection with the professional staff.

The focus of the work here presented is on the detection of potential anomalies in domestic activities, which are of key importance for professional staff to monitor the wellbeing of the person. Note this is not intended to be an alarm system detecting harmful situations. Instead, the detected anomalies must be interpreted by clinicians to assess symptoms and evolution of the MCI affecting the monitored patient.

In order to detect such anomalies, we exploit information coming from both the robot (RGB-D camera) and the AIS (domotic sensors), thus combining local and global activity information. The following section explains how these activity indicators are defined and used to identify potential anomalies.

\section{LocAl AND Global ACTIVITy LEVELS}

Two types of anomalies are usually considered in AAL: a) outlier detection and b) inappropriate behaviours. The first one is related to statistically uncommon behaviours, not observed in previous patterns. The second one is related to potentially harmful activities. The methods described next are particularly designed to detect the former type of anomalies as motion activity measures which are out of the expected levels.

To this end, we consider activity levels in two different contexts: local and global. Locally, we use the activity level detection with the robot's RGB-D camera described in Sec. IV-A. Local activity levels can be used to identify unusual motion behaviours of the user (e.g. running in the house) as observed by a robot. Global activity levels are defined according to the concept of normalized house entropy, which is described in Sec. IV-B. This provides information about the current activity situation of a single-occupant dwelling based on information from the domotic sensors (i.e. presence detectors).

Our system determines if a situation is an anomaly by combining these sources of information. The actual comparison between local and global activity levels is made by a dedicated MLN. MLNs enable automatic reasoning based on partial and incomplete observations [11]. At the same time, they offer a formal knowledge representation

\begin{tabular}{|l|l|l|l|}
\hline Feature & Parameter & Status & Activity Level \\
\hline Total & $\begin{array}{l}\text { Instant speed of the } \\
\text { person }\end{array}$ & $\begin{array}{l}\text { - stopped } \\
\text { - moving } \\
\text { - running }\end{array}$ & $\begin{array}{l}\text { - not active } \\
\text { - active } \\
\text { - highly active }\end{array}$ \\
\hline Head & $\begin{array}{l}\text { Instant speed of } \\
\text { head point }\end{array}$ & $\begin{array}{l}\text { - stopped } \\
\text { - moving }\end{array}$ & $\begin{array}{l}\text { - not active } \\
\text { - active }\end{array}$ \\
\hline Torso & $\begin{array}{l}\text { Instant speed of } \\
\text { shoulder points }\end{array}$ & $\begin{array}{l}\text { - stopped } \\
\text { - moving }\end{array}$ & - not active \\
& - active \\
\hline Body & $\begin{array}{l}\text { Standard deviation } \\
\text { of body volumes }\end{array}$ & $\begin{array}{l}\text { - stopped } \\
\text { - moving }\end{array}$ & - not active \\
& - active \\
\hline
\end{tabular}

TABLE I: Features used to detect human activity levels.

that can easily be used by a human expert to integrate new elements and describe other possible sources of anomalies. Our MLN implementation is described in detail in Sec. V.

\section{A. RGB-D Detection of Local Activity Levels}

Human activity levels are measured locally by the robot's RGB-D camera. Our human activity level detector covers four features: a) Total activity: refers to total motion of the person such as standing still, moving or rushing from one place to another; b) Torso activity: corresponds to torso motion, e.g. bending, turning around; c) Head activity: related to head movements, such as tilting left or right; d) Body activity: related to limbs motion, like head scratching, arms crossing, etc. A few examples of local activity detections are shown in Fig. 2.

The total activity level is based on the user's walking speed. This is obtained from a real-time multi-sensor people tracker [15], [16], which includes a laser-based legs detector and an RGB-D-based upper body detector. Both the detectors are fused using Bayesian filtering in order to estimate human location and velocity. The latter is then discretized as still / moving / running by applying two thresholds. The total activity level is categorized according to the discretized motion speed: if the same motion state persists longer than a given time interval (i.e. 5 seconds) the person is considered not active / active / highly active, respectively (see Table I).

Head, torso and body activity levels are derived from their speed and volume information. Speed and volume are calculated using the landmark point detection and body volume estimation techniques presented in [17]. Once head and shoulder motion speeds have been obtained, they are thresholded and classified as stopped or moving. Body activity levels are computed by categorizing volume changes of the body parts (i.e. stopped or moving), thresholding the standard deviation of the volume variations within a predefined time interval. As before, if the status of head, torso, or body remains the same for more than 5 seconds, the activity level of the person is deemed not active or active, respectively.

\section{B. Entropy-based Detection of Global Activity Levels}

The global activity levels are measured by our system using the concept of normalized house entropy. This metric is relatively straightforward and easy to obtain. Presence detectors trigger an event whenever motion is produced within their detection field. We have one presence sensor per relevant area, covering the entire floor-plan. With this 
setup, each sensor provides the amount of time $T\left(r_{i}\right)$ the user is moving in an specific location $r_{i}$. This information can be used to describe the probability $P\left(r_{i}\right)$ of some activity happening in a room:

$$
P\left(r_{i}\right)=\frac{T\left(r_{i}\right)}{\sum_{j} T\left(r_{j}\right)}
$$

Such probability provides some information about the current activity levels, but it is not a good metric to determine when the activity should be considered "abnormal". The statistics of such distribution indeed (e.g. variance) change with the order of the probabilities.

Instead, we propose an approach based on the entropy $H$, from information theory, to detect potentially abnormal situations. Entropy is invariant to probability permutations, as it describes the overall information contained in the distribution:

$$
H=-\Sigma_{i} P\left(r_{i}\right) \log _{2} P\left(r_{i}\right)
$$

When some motion activity takes place in the same room for most of the time, its probability distribution will be heavily concentrated in that location, thus low entropy levels will be expected. On the other hand, a uniformly distributed probability across many rooms generates high entropy levels, characterizing situations with a significant amount of activity.

We normalize this metric using the maximum possible entropy value, which occurs for discrete uniform distributions. In this case, the entropy is just given by the logarithm of the total number of possible outcomes. Therefore, the following normalized entropy $\hat{H}$ can be defined for a home environment with $R$ presence detectors and used to classify normal vs. abnormal situations:

$$
\hat{H}=\frac{H}{\log _{2} R}
$$

We use the 90th percentile criteria, suggested in [18], as an statistically meaningful indicator of anomaly. The normalized entropy values above this threshold will be considered indicative of abnormal activities. In the next section we describe how our MLN implementation combines this global activity metric with the previous local one.

\section{Markov Logic Network for Anomaly DETECTION}

Originally proposed by Richardson and Domingos in [11], MLNs combine both probabilistic and logical reasoning. A MLN consists of a set of weighted first-order logic formulas or clauses. The latter comprise the following elements:

- constants, which are possible objects in the domain;

- variables, describing a set of objects in a domain;

- functions, mapping relations between different objects;

- predicates, defining logical attributes or relationships over domain elements - they can be combined into more complex formulas using logical connectors.

Functions, variables and constants are called terms. If they do not contain variables, they are ground terms. A predicate that contains only ground terms is a ground predicate. When

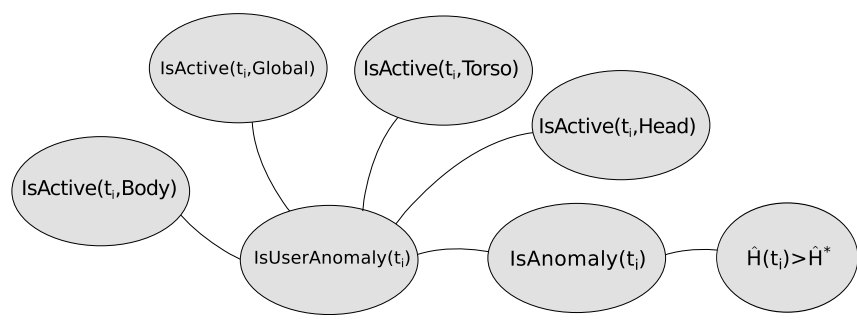

Fig. 3: Ground Markov network obtained by applying clauses A) and B) to the proposed features from the RGB-D activity level detector and the normalized entropy $\hat{H}$ at time $t_{i}$.

a logical value is assigned to all grounded predicates in a network, we have a possible world.

Using a finite set of constants applied over clauses in a MLN, we can define a Markov network. This network is an undirected graph with a binary node for every ground predicate. Ground predicates appearing in the same formula are linked, forming a clique. Depending on the given constants, each formula may have more than one clique associated to it. Each clique is assigned a logic feature and a weight, provided by the evaluation of the clause and its weight.

In essence, MLNs are compact representations of Markov networks. Once all clauses are applied over a domain, a MLN is grounded into a Markov network, and this network describes the probability of all possible combinations of grounded clauses. The latter form a knowledge base able to deal with incomplete and potentially contradictory evidence. This soft logic is possible due to weights that provide occurrence probabilities to logic clauses.

Using evidences, MLNs are able to produce Markov networks that describe the probability of all possible combinations of grounded clauses. We can then perform inference on Markov networks, usually by using approximate methods such as MC-SAT [10]. It is also possible to consider continuous values of evidence, instead of discrete ones only, using an extension called HMLN [10]. Thanks to the latter, our entropy metric can be included in a MLN clause.

Following these concepts, we define two clauses as evidence to combine our anomaly sources (normalized entropy $\hat{H}$ and RGB-D activity level). These two clauses describe a potentially anomalous situation at time $t_{i}$ in terms of each anomaly detector. The MLN outputs the likelihood of an anomaly fusing both the hypotheses, defined as follows:

A) one or more features describing the (local) activity levels from the robot's RGB-D camera are "active":

$$
\text { IsAnomaly }\left(t_{i}\right) \Rightarrow \bigcup_{\text {Feature }} \text { IsActive }\left(t_{i}, \text { Feature }\right)
$$

B) the (global) entropy from the presence detectors is higher than the 90th percentile entropy $\hat{H}^{*}$ :

$$
\text { IsAnomaly }\left(t_{i}\right) \wedge\left(\hat{H}\left(t_{i}\right) \geq \hat{H}^{*}\right)
$$

In our system, this MLN is implemented as a ROS service [19]. Fig. 3 shows a grounded MLN and the resulting 
graph for a time constant $t_{i}$. ROS is adopted as common framework for information exchange, enabling the robot to access the inference engine. The MLN service can be queried using evidence provided by any ROS source, including the normalized house entropy and the RGB-D activity levels. The output of the inference process is also available to any other node on the ROS network.

\section{EXPERIMENTS}

To evaluate the proposed system, we collected domotic sensor and RGB-D data for a week in an area of the ${\mathrm{L}-C A S^{7}}^{7}$ offices resembling a simple living environment with a kitchenette and a lounge space. The sensors were mostly concentrated in places where a rich set of activities are typically performed (entering, exiting, eating, drinking, resting, etc.). To facilitates the data recording, instead of the actual robot, we emulated its presence fixing an RGB-D camera on a tripod in front of the kitchenette. In this way, it was possible to collect long-term local information about user activities in this type of area, central to many ADLs.

\section{A. Domotic Sensors Dataset}

Twelve different people were working in these premises during the recordings. The sensors were placed at the entrance, in the kitchen, lounge area and workshop. Fig. 4 illustrates our sensors deployment and approximate area coverage.

The dataset contains more than 89,000 data entries from 37 different parameters recorded in two MongoDB collection snapshots. Our database storage offers great advantages for data management and manipulation compared to traditional log files and spreadsheet files. This is particularly important when we record long-term datasets. Here we need not only data storage, but also efficient and flexible querying methods. Using the MongoDB's query capabilities, we can freely retrieve any interval, sensor set and even combine data from other sources [20].

For the hardware, we used commercial ZWave wireless domotic sensors provided to ENRICHME by the Fibar Group $^{8}$. These sensors are easily deployable, widely available and have a long battery life. Their small size makes them also very unintrusive. All the recording and the processing was done by the same Intel NUC i7-5557U CPU @ 3.10GHz with 8 GB of RAM, running Linux OS Ubuntu 14.0464 bits.

\section{B. Human Activity Levels Results}

In this first part of the experiments, we consider only some local human activity levels detected on RGB-D data. A dedicated ROS module was implemented for the ENRICHME assistive robot in Fig. 1 and used for our kitchen camera. Since this is the central area of our testing environment, users often appear and perform various activities, which are detected also by the presence sensor.

\footnotetext{
${ }^{7}$ Lincoln Centre for Autonomous Systems - http:// lcas. Iincoln. ac.uk/

${ }^{8}$ http: //www.fibaro.com/
}

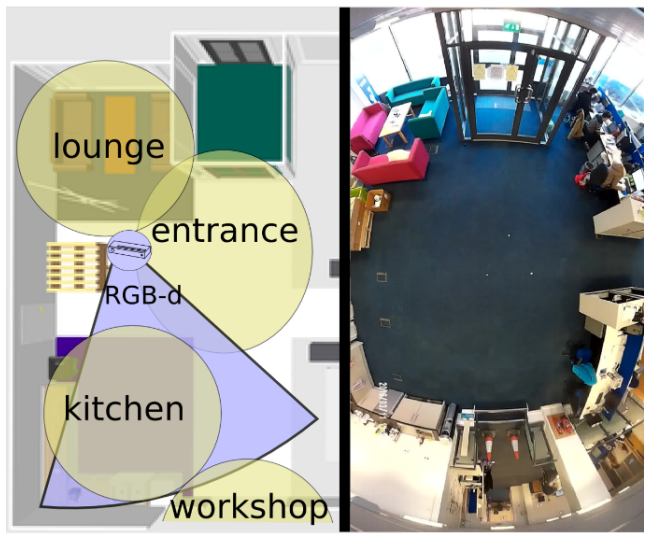

Fig. 4: Domotic sensors deployment in L-CAS.

The bottom graph in Fig. 5 shows the "Total" human activity level from RGB-D data, as defined in Sec. IV-A, recorded at the same time as the domotic sensors. Each level has one associated colour: red for Highly Active, green for Active and black for Not Active. The Active levels confirm that the users were performing some activities in the kitchen, like dish-washing or preparing coffee. The graph shows also a frequent detection of 'Highly Active' situations due to people quickly passing by the kitchen without stopping.

\section{Normalized Entropy Results}

We developed a ROS node that processed data from the presence detectors to calculate the normalized house entropy. Our entropy metric is only meaningful when applied to a set of binary sensors. On a single sensor, of course, it would not provide enough information about the user activities.

Fig. 5 presents a brief time lapse of some sensor data and the corresponding system entropy. It shows 10 minutes of sensor activations, detecting human motion in different locations. The thick red line in the top-three graphs represents the activation probability with respect to all the available sensors. The fourth graph illustrates the evolution of the normalized entropy, where the instantaneous values above

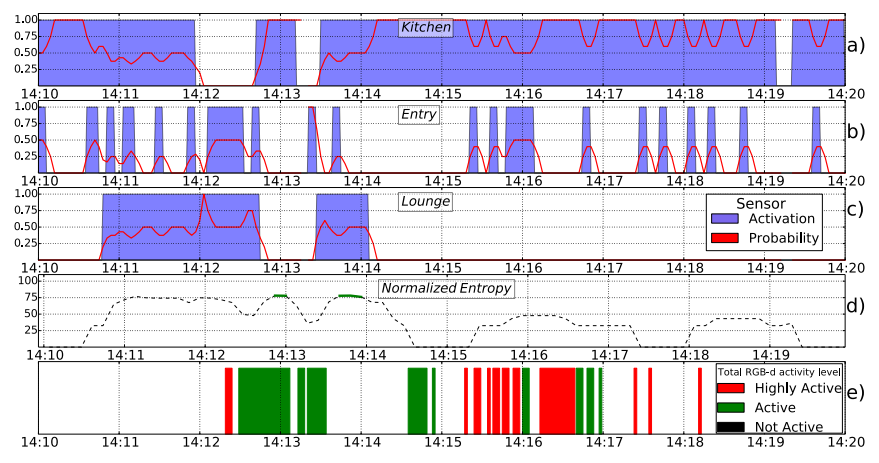

Fig. 5: a,b,c) Sensors activations and probabilities of (global) activities. Only three sensors are shown here, although the normalized entropy is computed for all the domotic sensors in the test environment. d) Normalized house entropy based on the domotic sensors. e) RGB-D detection of (local) activities. Different colours mean different activity levels. 
$75 \%$ are marked in green. In this case the $75 \%$ threshold corresponds to the 90th percentile criteria applied to our dataset, which is a common statistical indicator of anomaly detection [18].

As expected, the normalized entropy shows high values when all the sensors are active at the same time. The situation in the figure corresponds to events in the dataset where people were meeting after lunch for a coffee or to do some dish-washing. This type of activities generated high levels of activity detection in different areas simultaneously. In Sec. VI-D we will see how these can be considered an anomaly event (case \#4).

\section{Anomaly Detection Results}

Here we present the results using our MLN to detect potential anomalies of the activity levels. An anomaly event is reported when MLN anomaly probability is over $50 \%$. For comparison, we considered also a simple rule-based system implementing the same set of logic conditions defined in Sec. V, but without embedding them in a MLN. In practice, this rule-based detector produced binary outputs purely based on the simultaneous fulfilment of all rules.

Fig. 6 shows the output of both anomaly detectors (MLN vs. rule-based) and the respective snapshots of the detected events. Our ground truth is based on the observation of the RGB-D video stream by a human expert. Each event is numbered in the lower left corner of the image. Each one is also marked with a circle, showing which engine detected the event - blue for the rule-based and green for the MLN anomaly detector.

The events detected during the experiment are also summarized in Table II. In total, there were eight different events spotted by the anomaly detectors. The table shows how many users were currently visible by the RGB-D camera, which activities they were performing, and which detector classified them as anomalies. It shows also the activity level manually annotated as ground truth.

The graph in Fig. 6 illustrates the output of the anomaly detector during the experiment. The output of the rule-based anomaly detector is a binary function shown in blue. The output of the MLN anomaly detector is a probability value shown in red or green, depending if below or above a $50 \%$ confidence threshold, respectively.

The results show that the simple rule-based detector identifies six anomalies, numbered \#3 to \#8. During these events, all logical conditions were equally and simultaneously satisfied. The detector erroneously reported \#3 and \#8 as anomalies (false positives) because of some constant motion in the scene. It also missed two real anomalous events, \#1 and \#2 (false negatives), because the RGB-D detector failed to spot the high activity level, although the entropy from the domotic sensors was very high indeed.

The MLN detector, instead, missed the anomaly \#6, but also, correctly, the events \#3 and \#8. The detector, in this case, weighted the same logic clauses giving more importance to those in accordance with high entropy levels. Indeed, the events \#3, \#6 and \#8 had only moderate entropy

\begin{tabular}{|c|c|l|l|l|l|}
\hline \multirow{2}{*}{$\#$} & \multirow{2}{*}{ Users } & Activities & \multicolumn{3}{|c|}{ Anomaly Detection } \\
\cline { 4 - 6 } & 2 & $\begin{array}{l}\text { running, } \\
\text { having coffee }\end{array}$ & Yes & No* & Yes \\
\hline 2 & 3 & $\begin{array}{l}\text { walking, } \\
\text { kneeling, } \\
\text { running }\end{array}$ & Yes & No* & Yes \\
\hline 3 & 1 & cleaning & No & Yes* & No \\
\hline 4 & 2 & $\begin{array}{l}\text { walking, } \\
\text { dishwashing }\end{array}$ & No & Yes* & Yes* \\
\hline 5 & 3 & $\begin{array}{l}\text { moving fast, } \\
\text { walking, } \\
\text { dishwashing }\end{array}$ & Yes & Yes & Yes \\
\hline 6 & 3 & $\begin{array}{l}\text { walking, } \\
\text { kneeling, } \\
\text { cleaning }\end{array}$ & Yes & Yes & No* \\
\hline 7 & 1 & running & Yes & Yes & Yes \\
\hline 8 & 1 & making tea & No & Yes* & No \\
\hline
\end{tabular}

TABLE II: Detected anomalies. The users are only those visible by the camera. The ${ }^{\prime}{ }^{*}$ ' indicates a detection error.

levels, inducing the MLN to output anomaly probabilities below $50 \%$. Note that the influence of the entropy on the weights of the MLN clauses helped the latter to detect anomalies \#1 and \#2, which were missed instead by the simple rule-based detector.

Finally, we can see that both detectors erroneously detect \#4 as anomaly, although no relevant activity was happening at that time on the camera's view. However, the high entropy levels show that other people, not visible by the camera, were moving in the lounge and entrance area, triggering the anomaly events.

Overall, the MLN-based anomaly detector produced better results than the purely rule-based detector. It provided also a confidence value that can be further exploited in the future to improve the robustness of the anomaly detection.

\section{CONCLUSIONS AND Future Work}

We presented a new method to characterize the amount of activity performed in a human environment, using an information theoretic approach based on normalized entropy. The method is able to capture global activity levels from domotic sensors data, and it has been tested in real-world scenarios of natural human activities.

A new implementation of hybrid MLNs has been proposed to merge different sources of information, combining two different metrics of activity level to create an unsupervised anomaly detector. In particular, the normalized entropy is used as a new global indicator of user activities, while local activity levels are extracted from RGB-D data. This MLN-based approach has been tested successfully in real-world scenarios, showing its greater flexibility compared to a simpler rule-based detector, dealing also with situations of incomplete input data.

The dataset used in our experiments is publicly available for the research community. Not only it contains the presence detection information used in this work, but also a wider range of domotic sensor readings such as temperature, light, contact sensor states and electric consumption that can be exploited for extensions and alternative solutions.

In our future work we will include more expert knowledge in the MLN clauses, and consider additional sources of 

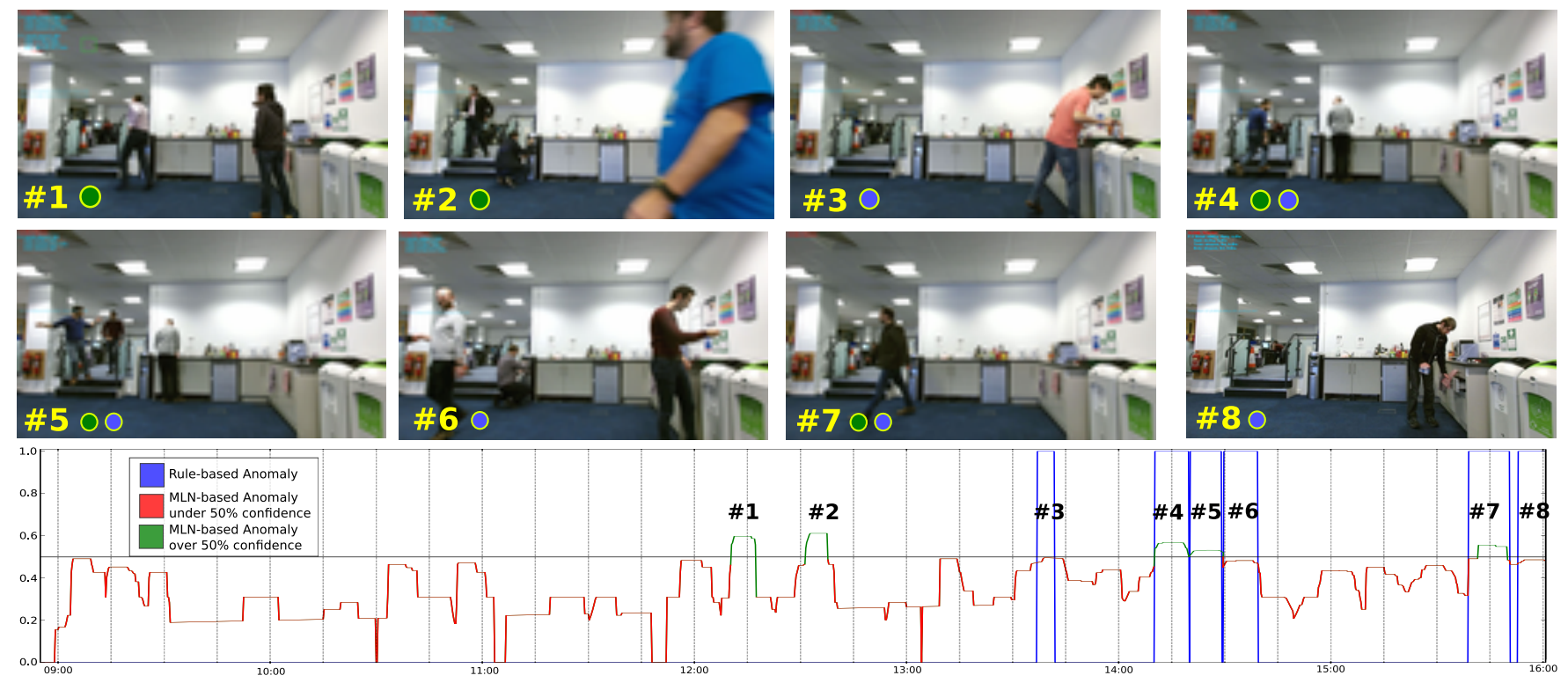

Fig. 6: Anomaly levels in L-CAS experiments and snapshots of detected anomalies (over 50\%).

information to improve the robustness of the anomaly detection in case of unexpected events or multiple people presence. Also, while the current system relies on a static MLN, new solutions will be explored to adapt dynamically to past evidence and patterns of human activities.

The proposed approach does not take into account the specific time of an activity, but only its duration and intensity. Since it can improve the detection of new anomaly types, our future work will consider also the time of the activities.

\section{ACKNOWLEDGMENT}

The research leading to these results has received funding from the EC H2020 Programme under grant agreement No. 643691, ENRICHME.

\section{REFERENCES}

[1] B. Rechel, E. Grundy, J. M. Robine, J. Cylus, J. P. MacKenbach, C. Knai, and M. McKee, "Ageing in the European Union," The Lancet, vol. 381, no. 9874, pp. 1312-1322, 2013.

[2] R. C. Petersen, B. Caracciolo, C. Brayne, S. Gauthier, V. Jelic, and L. Fratiglioni, "Mild cognitive impairment: a concept in evolution," Journal of Internal Medicine, vol. 275, no. 3, pp. 214-228, 2014.

[3] T. A. Bedrosian and R. J. Nelson, "Sundowning syndrome in aging and dementia: Research in mouse models," Experimental Neurology, vol. 243, pp. 67-73, 2013.

[4] A. Godfrey, M. Leonard, S. Donnelly, M. Conroy, G. ÓLaighin, and D. Meagher, "Validating a new clinical subtyping scheme for delirium with electronic motion analysis," Psychiatry Research, vol. 178, no. 1, pp. 186-190, 2010.

[5] D. Xu, Y. Yan, E. Ricci, and N. Sebe, "Detecting Anomalous Events in Videos by Learning Deep Representations of Appearance and Motion," Computer Vision and Image Understanding, vol. 156, pp. 117-127, 2016.

[6] D. Liciotti, T. Duckett, N. Bellotto, E. Frontoni, and P. Zingaretti, "Hmm-based activity recognition with a ceiling rgb-d camera," in ICPRAM - 6th International Conference on Pattern Recognition Applications and Methods, February 2017.

[7] J. Soulas, P. Lenca, and A. Thépaut, "Unsupervised discovery of activities of daily living characterized by their periodicity and variability," Engineering Applications of Artificial Intelligence, vol. 45, pp. 90-102, 2015.
[8] O. Aran, D. Sanchez-Cortes, M. T. Do, and D. Gatica-Perez, "Anomaly Detection in Elderly Daily Behavior in Ambient Sensing Environments," Human Behavior Understanding, pp. 51-67, 2016.

[9] H. Durrant-Whyte and T. C. Henderson, Multisensor Data Fusion. Berlin, Heidelberg: Springer Berlin Heidelberg, 2008, pp. 585-610.

[10] J. Wang and P. Domingos, "Hybrid Markov Logic Networks," in 23 National Conference on Artificial Intelligence, ser. AAAI'08, vol. 2. AAAI Press, 2008, pp. 1106-1111.

[11] M. Richardson and P. Domingos, "Markov logic networks," Machine learning, vol. 62, no. 1-2, pp. 107-136, 2006.

[12] P. Chahuara, A. Fleury, and M. Vacher, "Using Markov Logic Network for On-Line Activity Recognition from Non-visual Home Automation Sensors," Ambient Intelligence, pp. 177-192, 2012.

[13] B. Yuan and J. Herbert, "Context-aware hybrid reasoning framework for pervasive healthcare," Personal and Ubiquitous Computing, vol. 18, no. 4, pp. 865-881, 2014.

[14] L. Smirek, G. Zimmermann, and D. Ziegler, "Towards Universally Usable Smart Homes - How Can MyUI, URC and openHAB Contribute to an Adaptive User Interface Platform ?" in CENTRIC 2014 : The Seventh International Conference on Advances in Human-oriented and Personalized Mechanisms, Technologies, and Services, no. c, Nice, France, 2014, pp. 29-38.

[15] N. Bellotto and H. Hu, "Computationally efficient solutions for tracking people with a mobile robot: an experimental evaluation of Bayesian filters," Autonomous Robots, vol. 28, no. 4, pp. 425-438, 2010.

[16] C. Dondrup, N. Bellotto, F. Jovan, and M. Hanheide, "Real-time multisensor people tracking for human-robot spatial interaction," in Proc. of IEEE ICRA, Workshop on Machine Learning for Social Robotics, 2015.

[17] S. Cosar, C. Coppola, and N. Bellotto, "Volume-based human re-identification with rgb-d cameras," in Int. Conf. on Computer Vision Theory and Applications (VISAPP), 2017.

[18] D. Goldberg and Y. Shan, "The Importance of Features for Statistical Anomaly Detection," 7th USENIX Workshop on Hot Topics in Cloud Computing (HotCloud 15), pp. 1-6, 2015.

[19] M. Fernandez-Carmona and N. Bellotto, "On-line inference comparison with markov logic network engines for activity recognition in AAL environments," in IEEE International Conference on Intelligent Environments. IEEE, September 2016.

[20] T. Niemueller, G. Lakemeyer, and S. S. Srinivasa, "A generic robot database and its application in fault analysis and performance evaluation," in 2012 IEEE/RSJ International Conference on Intelligent Robots and Systems, Oct 2012, pp. 364-369. 